\title{
Agility Improvement through Cooperative Diversity in Cognitive Radio*
}

\author{
Ghurumuruhan Ganesan $\dagger$ and Ye (Geoffrey) Li \\ School of Electrical and Computer Engineering \\ Georgia Institute of Technology, Atlanta, Georgia 30332-0250
}

\begin{abstract}
In this paper, we illustrate the benefits of cooperation in cognitive radio. Cognitive (unlicensed) users need to continuously monitor spectrum for the presence of primary (licensed) users. We show that by allowing the cognitive radios operating in the same band to cooperate we can reduce the detection time and thus increase the overall agility. We first consider the case of two cognitive users and show how the inherent asymmetry in the network can be exploited to increase the agility. We then extend our protocol to study multi-user multi-carrier cognitive network. We compare our cooperation scheme with the non-cooperation scheme and derive expressions for agility gain. We show that our cooperation scheme reduces the detection time for the cognitive users by as much as $35 \%$.
\end{abstract}

Index Terms-cognitive radio, cooperative diversity, agility gain, detection time.

\section{INTRODUCTION}

The concept of software defined radio (SDR) was introduced in [1]. In SDR, the software embedded in a radio cell phone would define the parameters under which the phone should operate in real-time as its user moves from place to place. Cognitive radio $(\mathrm{CR})$ is even smarter than SDR. CR is a radio that is aware of and can sense its environment; learn from its environment; and perform functions that best serve its user. Since the cognitive (unlicensed) users are utilizing the licensed band, they must detect the presence of licensed (primary) users in a very short time and must vacate the band for the primary users. Thus one of the major challenges that confronts this technology is : how do the cognitive (unlicensed) radios sense the presence of the primary (licensed) user? One may expect this to be trivial but as shown in [2], there are fundamental limits to the detection capabilities of CR networks. In this paper, we show improvement in spectrum sensing capabilities through cooperation between individual cognitive users.

Cooperative networks achieve diversity gain by allowing the users to cooperate [3] [4]. Cooperative schemes with orthogonal transmission in a TDMA system have been recently proposed in [5] and [6]. It has been shown in [5] that two user single hop networks in which one of the user acts as a relay for the other, result in lower outage probabilities. In particular, it is shown that the amplify-and-forward (AF) protocol [5], in which the relay transmits the signal obtained from the

*This work was supported by NSF under Grant CCR-0121565 and the U. S. Army Research Laboratory under the Collaborative Technology Alliance Program, Cooperative Agreement DAAD19-01-20-0011.

$\dagger$ Corresponding Author: email : guru@ece.gatech.edu, phone/fax : 404385-3012/894-7883 transmitter without any processing, achieves full diversity. In this paper, we show that by allowing the users to cooperate under the AF protocol leads to much higher agility of the cognitive network. Also, we consider only the case where there are utmost two users per carrier and there is centralized scheduling to pair the users. Analysis of decentralized multiuser single-carrier networks can be found in [7].

The organization of the paper is as follows: In Section II, we formulate the problem for the simple two user case and show that the inherent asymmetry of the network can lead to faster detection with cooperation. We also describe the detector we shall use for detecting the presence of the primary user. In Section III, we develop expressions for asymptotic agility gain for the two user network and show improvement in agility. In Section IV, we extend our results to multi-user multi-carrier networks and state precise conditions under which agility gain is achieved. Finally, in Section V, present our conclusion.

\section{PRoblem Formulation}

We now describe the channel model of the system we use in the paper. We assume that all users experience Rayleigh fading that is independent from user to user. If a signal sent at the transmitter is $x$, the received signal $y$ is given by

$$
y=h x+w,
$$

where $h$ is the fading coefficient modelled as a complex Gaussian random variable and $w$ is additive white Gaussian noise. Unless otherwise mentioned all noise coefficients in this paper are assumed to be with zero-mean and unit-variance.

The main requirement of a cognitive radio architecture is to detect the presence of the primary or licensed users as quickly as possible. For that reason, the cognitive users should continuously monitor the spectrum for the primary (licensed) users. Consider the situation shown in Figure 1, where two cognitive radio users $U_{1}$ and $U_{2}$ operating in a fixed TDMA mode for sending data to a common receiver and a primary user starts using the band soon after. Then the two cognitive users need to vacate the band as soon as possible to make way for the primary user. However, the detection time becomes significant if one of the users is in the boundary of decodability of the primary user as shown in Figure 1. The signal received from the primary user is so weak that the cognitive user $U_{1}$ takes a lot of time to sense its presence. In this section, we describe our protocol which employs cooperation to reduce the detection time thereby improving the sensitivity of the cognitive receiver. Throughout the paper, we assume that : 
- The primary user location is known to both $U_{1}$ and $U_{2}$.

- Both the users when acting as relays have no transmit power constraint.

In [7], we incorporate power constraint for the relay user and study cooperative detection schemes for multi-user singlecarrier networks.

\section{A. Cooperation Scheme}

In Figure 2, we illustrate the protocol used by $U_{1}$ and $U_{2}$ to transmit data to some common receiver. We assume a slotted transmission system where $U_{1}$ and $U_{2}$ transmit in successive slots according to the AF protocol [5] in the same frequency band. Accordingly in time slot $T_{1}, U_{1}$ transmits and in $T_{2}, U_{2}$ relays the information of the previous slot. Unknown to both these users, a primary user has started using the band. This primary user must be detected as quickly as possible. At time slot $T_{1}, U_{1}$ transmits its data. Hence the signal received by $U_{2}$ in the time-slot $T_{1}$ is given by,

$$
y_{2}=\theta h_{p 2}+a h_{12}+w_{1},
$$

where $h_{p i}$ denotes the channel gain between the primary user and user $U_{i}$ and $h_{i j}$ denotes the channel gain between users $U_{i}$ and $U_{j}$. In (1), $a$ denotes the message sent from $U_{1}$ to the common receiver and $\theta$ indicates the presence of the primary user. Hence $\theta=1$ implies the presence of the primary user and $\theta=0$ implies its absence. In our cooperation protocol, we allow the user $U_{1}$ also to listen during the relay slot $T_{2}$. Thus the signal received by $U_{1}$ from $U_{2}$ during relay slot is given by

$$
y_{1}=\sqrt{\beta} h_{12}\left(a h_{12}+w_{1}+\theta h_{p 2}\right)+\theta h_{p 1}+w_{2},
$$

where $\beta$ refers to the scaling factor [5] used by $U_{2}$ to relay the information to the receiver. The scaling factor is usually chosen by $U_{2}$ to satisfy its power constraint [5]. In this paper, however, we assume that the relay user has no power constraint. Hence we can choose $\beta$ as we like. For simplicity, we choose

$$
\beta=\frac{1}{E\left\{\left|h_{12}\right|^{2}\right\}} .
$$

Throughout the paper, we assume that the channels are reciprocal symmetric, i.e., $h_{i j}=h_{j i}$. Thus, after the message component is cancelled from the received signal, the user $U_{1}$ is left with the signal

$$
Y=\theta H+W
$$

where $H=h_{p 1}+\sqrt{\beta} h_{p 2} h_{12}$ and $W=w_{1}+\sqrt{\beta} h_{12} w_{2}$. The detection problem can then be stated as:

Given the signal,

$$
Y=\theta H+W
$$

the detector decides on

$$
\mathcal{H}_{1}: \theta=1
$$

or

$$
\mathcal{H}_{0}: \theta=0 \text {. }
$$

We now utilize a simple energy detector [8] to show advantage of our proposed scheme.

\section{B. Energy Detector}

The reasons for choosing energy detector (ED) are twofold: (1) We want to show the effect of cooperation in cognitive networks. Hence the choice of detector is not critical. (2) We model the signal as a random variable with known power. Hence ED is optimal [8]. It can be shown that given $h_{12}=$ $h_{21}$, the random variables $H$ and $W$ in (2) are Rayleigh distributed with zero-mean and variances,

$$
\sigma_{H}^{2}=P_{1}+P_{2} h
$$

and

$$
\sigma_{W}^{2}=1+h
$$

respectively, where $h=\frac{\left|h_{12}\right|^{2}}{E\left\{\left|h_{12}\right|^{2}\right\}}, P_{1}=E\left\{\left|h_{p 1}^{2}\right|\right\}$ and $P_{2}=$ $E\left\{\left|h_{p 2}^{2}\right|\right\}$. Since $h_{12}$ is complex Gaussian, it is easily seen that $h$ is exponentially distributed with unity mean and variance. The ED forms the statistics

$$
T(Y)=|Y|^{2}
$$

and compares with a threshold $\lambda$ which is determined by some prespecified probability of false alarm $\alpha$. Define

$$
\varphi(t ; a, b)=\int_{0}^{\infty} e^{-h-\frac{t}{a+b h}} d h
$$

for positive $t, a$ and $b$. Also let $F_{i}(t)$ denote the cumulative density function (cdf) of the random variable $T$ under hypothesis $\mathcal{H}_{i}, i=0,1$. For $\mathcal{H}_{0}$ it can be shown that,

$$
\begin{aligned}
F_{0}(t) & =P\left(T(Y)>t \mid \mathcal{H}_{0}\right) \\
& =\int_{0}^{\infty} P\left(T(Y)>t \mid \mathcal{H}_{0}, h\right) f(h) d h \\
& =\int_{0}^{\infty} e^{-\frac{t}{1+h}} e^{-h} d h=\varphi(t ; 1,1) .
\end{aligned}
$$

Similarly, it can be shown that

$$
F_{1}(t)=\varphi\left(t ; P_{1}+1, P_{2}+1\right) .
$$

Hence, for a given probability of false alarm $\alpha$, we need to find the threshold $\lambda$ such that

$$
\varphi(\lambda ; 1,1)=\alpha .
$$

In fact $\lambda$ can be uniquely determined since $\varphi$ in (5) is strictly decreasing in $t$. Similarly the probability of detection by $U_{1}$ with cooperation from $U_{2}$ is found to be

$$
p_{c}^{(1)}=\varphi\left(\lambda ; P_{1}+1, P_{2}+1\right) .
$$

Compared to the non-cooperative case, the average SNR is increased by cooperation provided $P_{2}>P_{1}$. From (3) and (4), the instantaneous SNR is given by

$$
E\left\{\gamma_{c} \mid h\right\}=\frac{P_{1}+P_{2} h}{1+h} .
$$

Hence the average SNR in case of cooperation is given by

$$
\bar{\gamma}_{c}=\int_{0}^{\infty} E\left\{\gamma_{c} \mid h\right\} f(h) d h=\int_{0}^{\infty} \frac{P_{1}+P_{2} h}{1+h} e^{-h} d h
$$


For the non-cooperative case the average SNR is given by

$$
\bar{\gamma}_{n c}=P_{1} .
$$

Thus the SNR gain is,

$$
\bar{\gamma}=\frac{\bar{\gamma}_{c}}{\bar{\gamma}_{n c}}=\frac{P_{2}}{P_{1}}(1-F)+F,
$$

where

$$
F=\int_{0}^{\infty}(1+h)^{-1} e^{-h} d h<1 .
$$

In fact, $\bar{\gamma}-1=(1-F)\left(\frac{P_{2}}{P_{1}}-1\right)>0$ if $P_{2}>P_{1}$, which implies a SNR gain.

In Figure 3, we have shown the performance curve of the energy detector with and without cooperation. In the simulation, $P_{1}=1$ and $P_{2}=2.7$. Note that we assume that the relay user has no power constraint. Hence in the cooperative mode, the relay terminal adjusts its transmit power so that the channel gain between the users $U_{1}$ and $U_{2}$ is kept constant irrespective of the position of $U_{2}$. This serves as an upper bound on the performance of cooperation schemes. It can be seen that for the same probability of false alarm, we have higher detection probability through cooperation. This is the effect of cooperation in the network.

\section{AgILITY OF THE TWO USER COGNITIVE RADIO NETWORK}

In this section, we determine the time taken by the twouser network described in Section II to detect the presence of the primary user. We show that under the cooperation scheme described in Section II, the average detection time is reduced thus implying an increase in agility.

Let $\tau_{n}$ be the number of slots taken by user $U_{1}$ in a noncooperative network to detect the presence of the primary user. This detection time $\tau_{n}$ can be modelled as a geometric random variable, i.e.,

$$
\operatorname{Pr}\left\{\tau_{n}=k\right\}=\left(1-p_{n}^{(1)}\right)^{k-1} p_{n}^{(1)},
$$

where $p_{n}^{(i)}$ denotes the probability of detection by user $U_{i}$ in a single slot under the non-cooperation scheme. For the system model of our paper, it can be shown that

$$
p_{n}^{(1)}=\alpha^{\frac{1}{P_{1}+1}} \quad \text { and } \quad p_{n}^{(2)}=\alpha^{\frac{1}{P_{2}+1}} .
$$

The total time taken by both users to vacate the band can be shown to be

$$
T_{n}=2\left(\frac{1}{p_{n}^{(1)}}+\frac{1}{p_{n}^{(2)}}-\frac{1}{p_{n}^{(1)}+p_{n}^{(2)}-p_{n}^{(1)} p_{n}^{(2)}}\right) .
$$

If $p_{c}^{(i)}$ denotes the detection time for $U_{i}$ under the cooperation scheme described in Section II then from (7) we have

$$
p_{c}^{(1)}=\varphi\left(\lambda ; P_{1}+1, P_{2}+1\right),
$$

where $\lambda$ satisfies (6). It can be similarly shown that

$$
p_{c}^{(2)}=\varphi\left(\lambda ; P_{2}+1, P_{1}+1\right) .
$$

We have shown that if $P_{2}>P_{1}$ then

(1) $p_{c}^{(1)}>p_{n}^{(1)}$ and
(2) $p_{n}^{(2)}>p_{c}^{(2)}$.

Thus when the primary user location is known, we allow only $U_{2}$ to help $U_{1}$ and not vice-versa. In that case, the total average detection time of our cooperation scheme is given by

$$
T_{c}=\frac{2-\frac{p_{c}^{(1)}+p_{n}^{(2)}}{2}}{p_{c}^{(1)}+p_{n}^{(2)}-p_{c}^{(1)} p_{n}^{(2)}} .
$$

We define the agility gain of our cooperation scheme over the non-cooperation scheme when there are two users as

$$
\mu_{n / c}(2) \triangleq \frac{T_{n}}{T_{c}}
$$

Note that the agility gain $\mu(2)$ is a function of $P_{1}$ and $P_{2}$. However, without loss of generality we set $P_{1}=1$ and consider the agility gain as a function of $P_{2}$ alone. It can be shown that as $P_{2} \rightarrow \infty$, the asymptotic agility gain is given by,

$$
\mu_{n / c}^{\infty}(2)=\lim _{P_{2} \rightarrow \infty} \mu_{n / c}(2)=\frac{2}{\sqrt{\alpha}} .
$$

The non-cooperation scheme presented above assumes extremely selfish users. Hence cognitive user $U_{1}$ detects the presence of the primary user without the help of $U_{2}$. Also once detected $U_{1}$ vacates the band without informing $U_{2}$. Consider now a more practical situation where both the users get informed once either $U_{1}$ or $U_{2}$ detects the primary user. This is facilitated by transmitting the detection information to the common receiver which in turn informs the other cognitive users. Note that this is a partially cooperative network where the cognitive users still detect the primary user without any cooperation. In such a case, it can be shown that the time taken to vacate the band is given by,

$$
T_{p}=\frac{2-\frac{p_{n}^{(1)}+p_{n}^{(2)}}{2}}{p_{n}^{(1)}+p_{n}^{(2)}-p_{n}^{(1)} p_{n}^{(2)}} .
$$

The asymptotic agility gain in this case can be shown to be,

$$
\mu_{p / c}^{\infty}(2)=\lim _{P_{2} \rightarrow \infty} \frac{T_{p}}{T_{c}}=\frac{4}{3 \sqrt{\alpha}-\alpha} .
$$

In Figure 4 , we have plotted the agility gain $\mu_{n / c}(2)$ of a two user asymmetric network as a function of the asymmetry $P_{2}$ for different values of false alarm probability $\alpha$. As the network becomes more and more asymmetric, agility gain increases. From the figure, we note that for $\alpha=0.1$ as much as $35 \%$ reduction in detection time is achievable. Hence if one of the cognitive users is very close to the primary user, then we are guaranteed fast detection even if other user is very far away.

\section{Multiuser Cognitive network}

In this section we assume that there are $2 n$ cognitive users. The total bandwidth $B$ is equally divided into $n$ sub-bands each of bandwidth $\Delta_{n}=\frac{B}{n}$. There are two cognitive users working on each sub-band following the cooperation protocol described in Section II. It must be noted that the primary user, if present, uses the whole bandwidth $B$. If $P_{1}$ denotes the power received from the primary user at cognitive user $U_{1}$ when it uses the whole band, the signal power received at $U_{1}$ 
now is given by $P_{1}^{\prime}=\frac{P_{1}}{n}$. The noise power at $U_{1}$ is similarly scaled by a factor of $n$. Suppose that users $U_{1}$ and $U_{2}$ form a link in a particular sub-band with $U_{2}$ acting as a relay for $U_{1}$. The detection problem for that sub-band is identical to (2) except that

and

$$
\sigma_{H}^{2}=\frac{1}{n}\left(P_{1}+P_{2} h\right)
$$

$$
\sigma_{W}^{2}=\frac{1}{n}(1+h)
$$

where, as before, $h$ denotes the scaled channel gain between the pair of users. Thus the detection problem for multi-carrier networks is similar to that of single-carrier networks except for a scaling factor of $n$. Hence for a given probability of false alarm $\alpha$ we need to find the threshold $\lambda_{n}$ such that

$$
\varphi\left(\lambda_{n} ; \frac{1}{n}, \frac{1}{n}\right)=\alpha \text {. }
$$

It can be easily shown that $\lambda_{n}=\frac{\lambda}{n}$ where $\lambda$ is given by (6). Thus for example, the probability of detection by $U_{1}$ with cooperation from $U_{2}$ is found to be

$$
p_{c}^{(1)}=\varphi\left(\lambda_{n} ; \frac{P_{1}+1}{n}, \frac{P_{2}+1}{n}\right)=\varphi\left(\lambda ; P_{1}+1, P_{2}+1\right) .
$$

It follows that the detection probability for a particular user is the same independent of the bandwidth the user is occupying.

It can be seen that the probability that the cognitive users in a sub-band detect the primary user under the cooperation scheme and the non-cooperation scheme are given by

$$
p=p_{c}^{(1)}+p_{n}^{(2)}-p_{c}^{(1)} p_{n}^{(2)}
$$

and

$$
p^{\prime}=p_{n}^{(1)}+p_{n}^{(2)}-p_{n}^{(1)} p_{n}^{(2)}
$$

respectively. Since $U_{2}$ acts as a relay for $U_{1}$ and not viceversa, it is obvious that from the discussion in the Section III, $p>p^{\prime}$. Note that there are $n$ sub-bands occupying the band. For $k=1,2, \ldots, n$, let $p_{k}$ and $p_{k}^{\prime}\left(<p_{k}\right)$ denote the probability of detection of the $k^{t h}$ sub-band to detect the presence of the primary user with and without cooperation respectively. As before we shall consider two cases of non-cooperation:

(i) All the $n$ sub-bands detect and vacate independently of other sub-bands (totally non-cooperative).

(ii) The sub-bands detect the primary user independently, but the first sub-band to detect the primary user informs the other sub-bands through a common base station (partially cooperative).

In case of (ii), it can be shown that the average number of slots taken for detection under cooperation and non-cooperation are given, respectively, by

$$
T_{c}(n)=\frac{1}{1-\prod_{k=1}^{n}\left(1-p_{k}\right)}
$$

and

$$
T_{p}(n)=\frac{1}{1-\prod_{k=1}^{n}\left(1-p_{k}^{\prime}\right)} .
$$

Define the agility gain to be

$$
\mu_{p / c}(n)=\frac{T_{p}(n)}{T_{c}(n)}
$$

It can be easily shown that for all $n$

$$
\mu_{p / c}(n)>1
$$

and

$$
\lim _{n \rightarrow \infty} \mu_{p / c}(n)=1 .
$$

Hence for all finite cognitive user population we have agility gain, though, asymptotically, there is little difference between partial cooperation and total cooperation.

For the totally non-cooperative network, we shall assume that the probability of detection is equal for all the sub-bands in consideration. Though this is strictly not true, we make this simplifying assumption because we are concerned not in exploiting the asymmetry of the network but the presence of multiple cognitive users. Let $p^{\prime}=1-q^{\prime}$ denote the detection probability for all the $n$ sub-bands under the totally noncooperation scheme. It can be shown that the total time taken for detection is then given by

$$
T_{n}(n)=\sum_{k=1}^{n}\left(\begin{array}{l}
n \\
k
\end{array}\right)(-1)^{k+1} \frac{1}{1-\left(q^{\prime}\right)^{k}} .
$$

As before, we have defined the agility gain to be

$$
\mu_{n / c}(n)=\frac{T_{n}(n)}{T_{c}(n)}
$$

and have proved that

$$
\lim _{n \rightarrow \infty} \frac{\mu_{n / c}(n)}{\ln n}=\Theta\left(-\frac{1}{\ln (1-\alpha)}\right) .
$$

Thus the agility gain grows as $\ln n$. In Figure 5, we have plotted the agility gain $\mu_{n / c}(n)$ of our multi-user multi-carrier cooperation scheme for various values of the probability of false alarm, $\alpha$. Since $\alpha$ is very small, $\ln (1-\alpha) \approx-\alpha$, we see that the agility gain curves are well approximated as $\mu_{n / c}(n) \approx \frac{\ln n}{\alpha}$ for large $n$.

\section{CONClusion}

In this paper, we have shown the benefits of cooperation in increasing the agility of cognitive radio networks. We first considered a simple two user cooperative cognitive network and showed improvement in agility by exploiting the inherent asymmetry. We then extended our cooperation scheme to multi-user multi-carrier networks and stated precise conditions under which agility gain is achieved. We analyzed two cooperative schemes employing varying degrees of cooperation : (1) totally non-cooperative, where each user detects the primary user and vacates without informing the other users and (2) partially cooperative, where the first user to detect the primary user informs the other cognitive users through a common base station. We found that for multi-carrier networks, asymptotically there is no difference between the cooperation scheme described in Section II and partially cooperative scheme. Compared to totally non-cooperative scheme, however, the agility gain of our cooperation scheme was found to be $\Theta(\ln n)$ where $n$ is the number of sub-bands. 


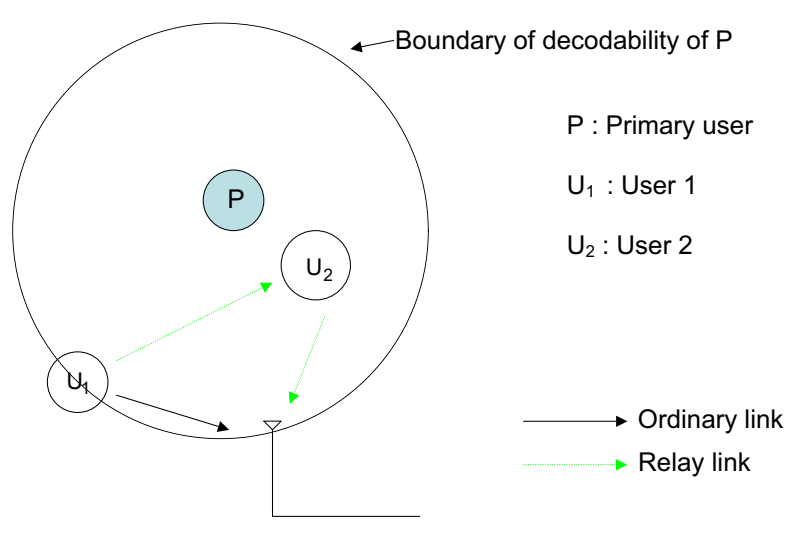

Fig. 1. Cooperation in cognitive network

\begin{tabular}{|l|l|l|l|l|l|l}
\hline $\mathrm{U}_{1} \mathrm{Tx}$ & $\mathrm{U}_{2}$ Relay & $\mathrm{U}_{2} \mathrm{Tx}$ & $\mathrm{U}_{1}$ Relay & $\cdots$ & $\underset{\text { time }}{\longrightarrow}$
\end{tabular}

Fig. 2. Relay protocol used

\section{REFERENCES}

[1] I. J. Mitola, "Software radios: Survey, critical evaluation and future directions," IEEE Aerosp. Electron. Syst. Mag., vol. 8, pp. 25-31, Apr. 1993.

[2] A. Sahai, N. Hoven and R. Tandra, "Some fundamental limits in cognitive radio," in Allerton Conf. on Commun., Control and Computing, Oct. 2004.

[3] A. Sendonaris, E. Erkip and B. Aazhang, "User cooperation in diversity - Part I: System description," IEEE Trans. Commun., vol. 51, pp. 19271938, Nov. 2003.

[4] — , "User cooperation in diversity - Part II: Implementation aspects and performance analysis," IEEE Trans. Commun., vol. 51, pp. 1939-1948, Nov. 2003.

[5] J. N. Laneman and D. N. C. Tse, "Cooperative diversity in wireless networks: Efficient protocols and outage behaviour," IEEE Trans. Inform. Theory, vol. 50, pp. 3062-3080, Dec. 2004

[6] J. N. Laneman and G. W. Wornell, "Distributed space-time coded protocols for exploiting cooperative diversity in wireless networks," IEEE Trans. Inform. Theory, vol. 49, pp. 2415-2425, Oct. 2003.

[7] G. Ganesan and Y. G. Li, "Cooperative spectrum sensing in cognitive radio networks," in IEEE DYSPAN 2005, St. Louis, Missouri, Nov. 2005, accepted for publication.

[8] H. V. Poor, An Introduction to Signal Detection and Estimation, 2nd ed. Springer-Verlag, 1994

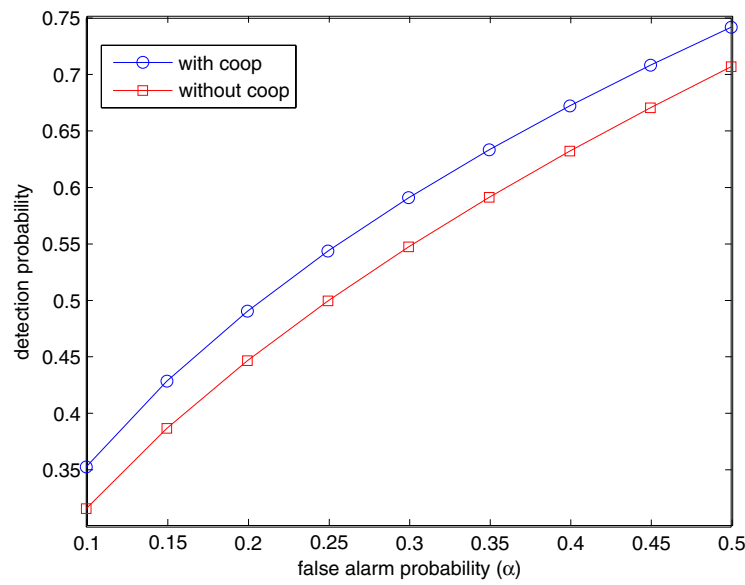

Fig. 3. Performance Curves

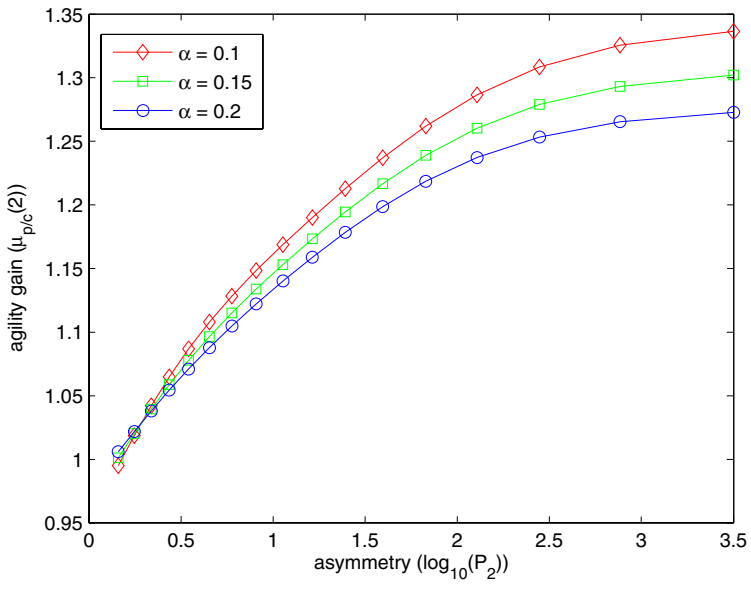

Fig. 4. Agility gain in single carrier two user asymmetric networks

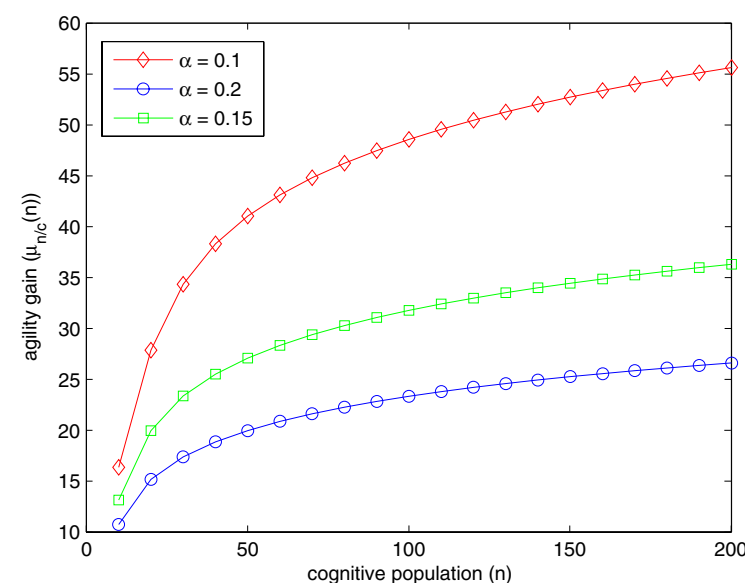

Fig. 5. Agility gain in multi-carrier multi-user symmetric networks 